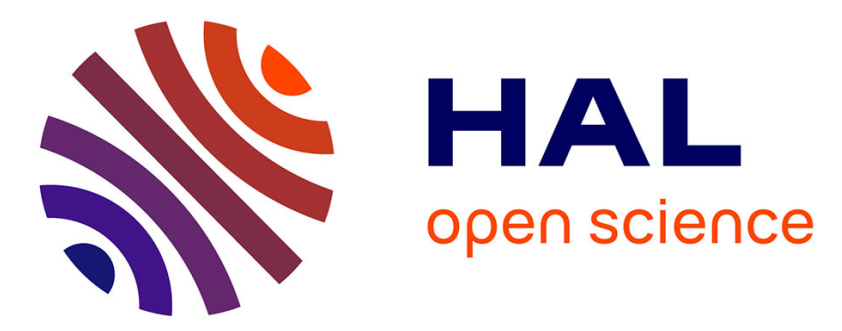

\title{
Ab initio study of oxygen point defects on tungsten trioxide surface
}

Caroline Lambert-Mauriat, Vincent Oison, Lama Saadi, Khalifa Aguir

\section{To cite this version:}

Caroline Lambert-Mauriat, Vincent Oison, Lama Saadi, Khalifa Aguir. Ab initio study of oxygen point defects on tungsten trioxide surface. Surface Science: A Journal Devoted to the Physics and Chemistry of Interfaces, 2012, 606 (1-2), pp.40 - 45. 10.1016/j.susc.2011.08.018 . hal-01730711

\section{HAL Id: hal-01730711 \\ https://hal.science/hal-01730711}

Submitted on 13 Mar 2018

HAL is a multi-disciplinary open access archive for the deposit and dissemination of scientific research documents, whether they are published or not. The documents may come from teaching and research institutions in France or abroad, or from public or private research centers.
L'archive ouverte pluridisciplinaire HAL, est destinée au dépôt et à la diffusion de documents scientifiques de niveau recherche, publiés ou non, émanant des établissements d'enseignement et de recherche français ou étrangers, des laboratoires publics ou privés. 


\title{
Ab initio study of oxygen point defects on tungsten trioxide surface
}

\author{
Caroline Lambert-Mauriat, Vincent Oison, Lama Saadi and Khalifa Aguir \\ Aix-Marseille Université, IM2NP \\ CNRS, IM2NP (UMR 6242)

\begin{abstract}
Faculté des Sciences et Techniques, Campus de Saint-Jérôme, Avenue Escadrille Normandie Niemen - Case 142, F-13397 Marseille Cedex 20, France
\end{abstract}

\begin{abstract}
The gas response of tungsten trioxide $\left(\mathrm{WO}_{3}\right)$ based sensors strongly depends on the surface properties. Reconstructed surfaces and oxygen point defects at the surface of the monoclinic $\mathrm{WO}_{3}$ are studied using a self-consistent scheme based on first-principle. The oxygen vacancy is found to be the predominant defect independently of the oxygen partial pressure. Indeed, under rich oxygen atmosphere the formation enthalpies are found to be $1.45 \mathrm{eV}$ in LDA (1.28 eV in GGA) for the oxygen vacancy instead of $2.70 \mathrm{eV}(2.42 \mathrm{eV})$ for the oxygen adatom. When the oxygen partial pressure is lowered, the oxygen vacancy formation enthalpy decreases and becomes exothermic under very O-poor condition (-1.65 eV in LDA and $-1.36 \mathrm{eV}$ in GGA). On the other hand, the formation enthalpy of an oxygen adatom rises. Finally, the oxygen vacancy formation acts as a $n$-doping by introducing negative charge carriers at the bottom of the conduction band. All these results can be very helpful in order to explain the electrical resistivity measurements.
\end{abstract}

Email address: caroline.mauriat@im2np.fr (Caroline Lambert-Mauriat) 
Keywords: $\mathrm{WO}_{3}$, gas sensor, DFT calculations, oxygen point defects, surface properties, oxygen vacancy $n$-doping

\section{Introduction}

Tungsten trioxide $\left(\mathrm{WO}_{3}\right)$ is a wide band-gap $\left(\mathrm{E}_{g}=2.62 \mathrm{eV}[1]\right.$ or $\left.2.7 \mathrm{eV}[2]\right)$ n-type semiconductor. It shows numerous polymorphs [3] derived from the $\mathrm{ReO}_{3}$ structure which involves a cubic array of corner-shared octahedra. At room temperature the $\gamma-\mathrm{WO}_{3}$ monoclinic phase of space group $P 2_{1 / n}$ is observed. For several years $\mathrm{WO}_{3}$ has been used as a sensitive element in gas sensor devices, such as ammonia $\mathrm{NH}_{3}[4,5]$ or ozone $\mathrm{O}_{3}[6,7,8]$. Detection relies on phenomena of adsorption and catalysis, which modify the electrical resistivity. For ozone detection, models based on adsorption/desorption have been developed [9] in which adsorption energy is a crucial parameter. Unlike $\mathrm{WO}_{3}, \mathrm{SnO}_{2}$ - well-known and commonly used in commercial gas sensor - has already been studied theoretically. Indeed the interaction of oxygen with $\mathrm{SnO}_{2}$ (110) surface has been studied by DFT calculations ten years ago [10]. It has been underlined that oxygen vacancies play a major role in the adsorption of neutral molecules since oxygen cannot be exothermically adsorbed from the gas phase on the stoichiometric surface.

Tungsten trioxide is supposed to be similar to other oxides like tin oxide for which point defects of oxygen play a major role in the ozone adsorption by modifying the charge density near the surface. Furthermore the oxygen vacancies are expected to be the preferential sites for the chemical reduction of molecules, whereas oxygen adatom should be the preferential sites for the oxidation of molecules. Due to the large anisotropy of the W-O bonds fol- 
lowing the $\mathbf{c}$ crystallographic direction, the (001) plane is the easiest one for cleaving the crystal. By consequence the (001) reconstructed surfaces are found to be the most stable for the $\gamma-\mathrm{WO}_{3}$ monoclinic phase [11]. In this paper we address the following question: what is the effect of the $\mathrm{O}$ point defects on the electronic structure of $\mathrm{WO}_{3}$, in particular on the doping? In addition, we investigate the key-role of the oxygen partial pressure on the defect properties.

This article is organized as follows: in section 2 computational details are reported. The $(1 \times 2),(2 \times 1)$ and $(\sqrt{2} \times \sqrt{2}) \mathrm{R} 45^{\circ}$ reconstructions of the stoichiometric surface are studied in section 3 . All calculations are performed using supercells consisting in a slab of eight atomic planes and vacuum layer in the $\mathbf{c}$ direction. The surfaces are obtained by cleaving the crystal perpendicularly to the $\mathbf{c}$ cristallographic axis and by using the cell parameters obtained in our previous work [12]. The convergence of the surface energy with respect to the size of the slab is presented and results obtained using the local density approximation (LDA) and the generalized gradient approximation (GGA) are compared. In section 4, the formation energy of an O-vacancy (or an O-adatom) is investigated. In particular, the induced modifications in the electronic density of states (DOS) are analyzed. In addition a simple thermodynamic model is introduced in order to take into account the effect of the oxygen partial pressure. Finally, our results are used to interpret the experimental measurements of the electrical resistivity under different oxygen pressures. 


\section{Computational details}

DFT calculations are carried out using the SIESTA code [13, 14]. The exchange-correlation potential is treated either within the local density approximation (LDA) using the Ceperley-Alder functional [15] or within the generalized gradient approximation (GGA) using the Perdew-Burke-Ernzerhof functional [16]. In all cases core electrons are treated within the frozen core approximation using norm-conserving Troullier-Martins [17] pseudopotentials. Nonlinear core corrections are included for $\mathrm{W}$ pseudopotentials. The wave-function of the valence electrons $\left(2 s^{2} 2 p^{4}\right.$ for $\mathrm{O}$ and $6 s^{2} 5 d^{4}$ for $\left.\mathrm{W}\right)$ is expanded on a localized basis set consisting in finite-range pseudo-atomic orbitals [18]. A double- $\zeta$ basis set is used for all the atoms: each atomic valence state is described by two localized wave functions. In order to allow polarization, $3 d$ and $6 p$ orbitals are included for $\mathrm{O}$ and $\mathrm{W}$ species, respectively. The optimized basis set for $\mathrm{W}$ and $\mathrm{O}$ atoms is taken from our previous work on bulk properties [12]. All calculations are performed using supercell consisting in slab of several atomic planes and vacuum of $15 \AA$ approximativelly along the $\mathbf{c}$ direction. The slab is built from the atomic structure of the monoclinic phase as shown on figure 1. The lattice parameters obtained from LDA (GGA) calculations are $a=7.24 \AA, b=7.45 \AA, c=7.61 \AA$ and $\beta=90.5^{\circ}\left(a=7.48 \AA, b=7.67 \AA, c=7.85 \AA\right.$ and $\left.\beta=90.4^{\circ}\right)$. Experimental values determined by neutron diffraction are $a=7.31 \AA, b=7.54 \AA$, $c=7.69 \AA$ and $\beta=90.9^{\circ}[19]$. Perpendicularly to the surface, mixed WO planes alternate with pure O planes along the [001] direction,. When convergence is studied as a function of the thickness of the slab, the periodicity along $\mathbf{a}$ and $\mathbf{b}$ directions parallel to the surface remains the same as in the 


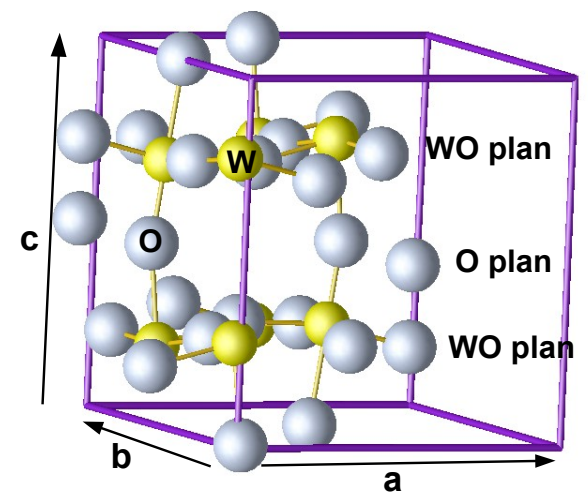

Figure 1: Atomic structure of the monoclinic phase (GGA calculations). Tungsten atoms are represented by yellow spheres and oxygen atoms by grey spheres. WO and O planes are pointed out.

bulk. In this case the $\mathbf{k}$-point sampling consists in a $8 \times 8 \times 1 \mathbf{k}-$-mesh: eight $\mathbf{k}-$ points along $\mathbf{a}^{*}$ and $\mathbf{b}^{*}$ and one along $\mathbf{c}^{*}$.

To decrease the oxygen defect (vacancy or adatom) concentration at the surface, the unit cell is doubled in the $\mathbf{a}$ and $\mathbf{b}$ directions. This leads to a defect concentration of $1 / 8$. In this case a $4 \times 4 \times 1 \mathbf{k}$-mesh grid is used in order to ensure an equivalent $\mathbf{k}$-point density. Finally, to keep the size of the basis set constant, a so-called ghost atom is used when an oxygen atom is removed or added. In all calculations, atomic positions are relaxed until the forces on each atom converge to less than $0.02 \mathrm{eV} / \AA$.

\section{Surface properties}

\subsection{Study of surface reconstructions}

In this section, we present results obtained from ab initio calculations on slabs consisting in two monoclinic unit cells superposed along the $\mathbf{c}$ cristal- 
lographic direction. Each of them contains 64 atoms and consists in $4 \mathrm{WO}$ atomic planes alternating with 4 pure $\mathrm{O}$ planes. The reconstruction being necessary to ensure both the stoichiometry and the neutrality of the surface, one oxygen out of two has to be removed. Among the five reconstructions reported for the cubic phase [11], only the three less energetically expensive ones are here considered. They correspond to the $(1 \times 2),(2 \times 1)$ and $(\sqrt{2} \times \sqrt{2}) \mathrm{R} 45^{\circ}$ reconstructions, as represented in figure 2 . In the case of the $(\sqrt{2} \times \sqrt{2}) \mathrm{R} 45^{\circ}$ reconstruction, the oxygen atoms form rows along the [110] direction, while in the case of the $(1 \times 2)$ and $(2 \times 1)$ reconstructions, they form lines, respectively, along the [100] and the [010] directions. The

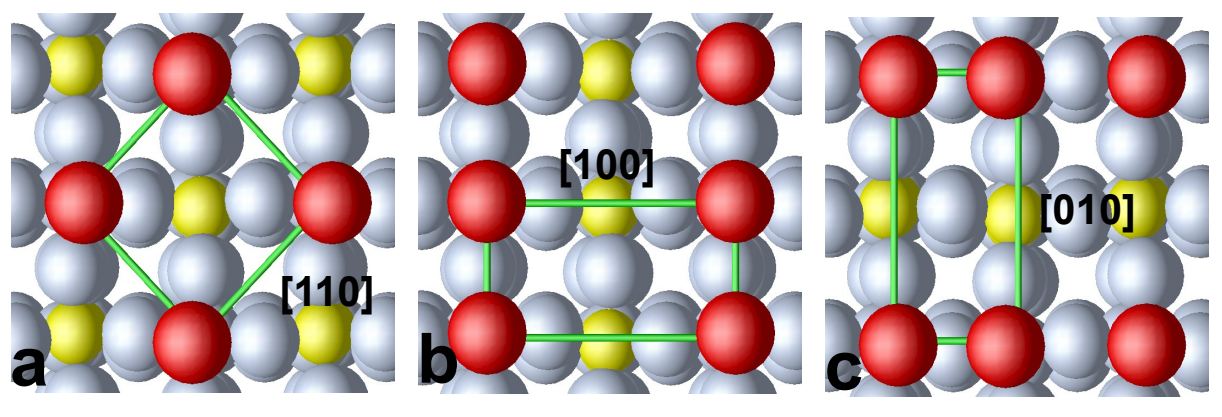

Figure 2: Top view of O-terminated surface for the reconstructions: (a) $(\sqrt{2} \times \sqrt{2}) \mathrm{R} 45^{\circ}$, (b) $(2 \times 1)$ and (c) $(1 \times 2)$. The surface oxygen atoms are represented by red spheres, tungsten and other oxygen atoms are represented by yellow and grey spheres, respectively. The atomic positions are obtained from GGA calculations.

surface energy, $\sigma$, is then given by:

$$
\sigma=\frac{1}{2 S}\left[E_{\text {slab }}\left(W_{n} O_{3 n}\right)-E_{b u l k}\left(W_{n} O_{3 n}\right)\right],
$$

where $E_{\text {slab }}\left(W_{n} O_{3 n}\right)$ is the total energy of the relaxed slab, $E_{b u l k}\left(W_{n} O_{3 n}\right)$ is the total energy of the bulk, $n$ is the number of $\mathrm{W}$ atoms in the supercell and 
$S=|\mathbf{a} \times \mathbf{b}|$ is the surface unit cell $\left(S=57.36 \AA^{2}\right.$ in GGA and $S=53.98 \AA^{2}$ in LDA). In equation (1) the presence of the one half prefactor denotes that two surfaces are created due to the periodic boundary conditions.

\begin{tabular}{ccc}
\hline \hline Reconstruction & LDA & GGA \\
\hline$(\sqrt{2} \times \sqrt{2})$ R45 & 41.3 & 21.0 \\
$(2 \times 1)$ & 44.8 & 23.2 \\
$(1 \times 2)$ & 47.6 & 25.9 \\
\hline \hline
\end{tabular}

Table 1: Surface energy, $\sigma$, given in $\mathrm{meV} / \AA^{2}$ of the $(\sqrt{2} \times \sqrt{2}) \mathrm{R} 45^{\circ},(2 \times 1)$ and $(1 \times 2)$ reconstructions. Comparison between LDA and GGA.

For the three reconstructions, the values of the surface energy obtained from the LDA are nearly two times as large as the ones obtained from the GGA. This difference can be rationalized by significant effects of structural relaxation, which convert long $\mathrm{W}-\mathrm{O}$ bonds into short ones and vice-versa along the $\mathbf{c}$ direction. Let us note that surface energies (LDA or GGA) obtained without any relaxation only display a difference of less than 10 percent. For $(\sqrt{2} \times \sqrt{2}) \mathrm{R} 45^{\circ}$ reconstruction, the unrelaxed surface energies amount to $227.6 \mathrm{meV} / \AA^{2}$ in LDA and $207.5 \mathrm{meV} / \AA^{2}$ in GGA. Nevertheless in all cases the $(\sqrt{2} \times \sqrt{2}) \mathrm{R} 45^{\circ}$ reconstruction is energetically the most favorable (Table 1). Therefore, the values of $\sigma$ obtained for the $(2 \times 1)$ and the $(1 \times 2)$ reconstructions - respectively $23.2 \mathrm{meV} / \AA^{2}(44.8)$ and $25.9 \mathrm{meV} / \AA^{2}$ (47.6) in the GGA (LDA) - are a little larger than the ones obtained for $(\sqrt{2} \times \sqrt{2}) \mathrm{R} 45^{\circ}$ reconstruction, $20.9 \mathrm{meV} / \AA^{2}$ (41.3). The GGA values are in good agreement with those obtained by Yakovkin and Gutowski on the cubic phase $\left(22 \mathrm{meV} / \AA^{2}\right)$ and on the monoclinic $\epsilon$ phase $\left(19 \mathrm{meV} / \AA^{2}\right)$, us- 
ing the VASP code [20]. Semi-empirical calculations performed by Oliver and co-workers [11] have given the same order in surface energies for these three reconstructions. Experimentally, STM and LEED observations made by Dixon and co-workers [21] have shown that the $(\sqrt{2} \times \sqrt{2}) \mathrm{R} 45^{\circ}$ reconstruction is usually observed on weakly reduced surfaces and, consequently, this coroborates our numerical results.

\subsection{Effect of the slab thickness}

The effect of the slab thickness on the value of $\sigma$ is studied by increasing the number of WO atomic planes along the [001] direction. The slabs are noted $\mathrm{W}_{n} \mathrm{O}_{3 n}$, where $n$ is the number of $\mathrm{W}$ atoms per cell. In all calculations, the translation vectors parallel to the surface correspond to the $\mathbf{a}$ and $\mathbf{b}$ vectors of the monoclinic unit cell. The thickness of the slab varies from 3 WO planes $\left(\mathrm{W}_{12} \mathrm{O}_{36}\right.$ supercell) to $10 \mathrm{WO}$ planes $\left(\mathrm{W}_{40} \mathrm{O}_{120}\right)$.

\begin{tabular}{cccc}
\hline \hline$n$ & number of WO planes & LDA & GGA \\
\hline 12 & 3 & 40.2 & 20.2 \\
16 & 4 & 41.3 & 21.0 \\
24 & 6 & 42.6 & 21.3 \\
32 & 8 & 43.9 & 21.4 \\
40 & 10 & 44.1 & 21.8 \\
\hline \hline
\end{tabular}

Table 2: Surface energy, $\sigma$, given in $\mathrm{meV} / \AA^{2}$ for the $(\sqrt{2} \times \sqrt{2}) \mathrm{R} 45^{\circ}$ reconstruction as a function of the number of WO planes within the slab. Comparison between LDA and GGA calculations.

As shown in table 2, the values of $\sigma$ slightly increase with respect to the number of WO planes. From 3 to 10 WO planes, the value of $\sigma$ increases by 
$9 \%$ (LDA) and $5 \%$ (GGA). To conclude the $\mathrm{W}_{16} \mathrm{O}_{48}$ slab containing $4 \mathrm{WO}$ planes seems to be a good compromise between the size of the slab and the precision of the calculations.

\subsection{Electronic structure}

In comparison with the electronic structure of the bulk, the band gap of the $(\sqrt{2} \times \sqrt{2}) R 45^{\circ}$ surface is reduced by a factor two. Let us recall that the band gap is usually underestimated, both in GGA than in LDA, by a factor of 2.5 (1 eV in the bulk instead of $2.5 \mathrm{eV}$ experimentally [12]). Similarly the atomic Mulliken charges do not depend on the choice of the exchangecorrelation functional. In comparison with oxygen in the bulk, the negative charge carried by the surface $\mathrm{O}$ atoms is two times lower: $-0.26 e$ instead of $-0.50 e\left(e=1.6 \times 10^{-19} \mathrm{C}\right)$. The positive charge carried by the surface $\mathrm{W}$ atoms is slightly lower than its bulk value: $+1.63 e$ instead of $+1.68 e$ within the GGA and $+1.52 e$ instead of $+1.56 e$ within the LDA ones. These results are consistent with the fact that one $\mathrm{W}-\mathrm{O}$ bond is broken for each $\mathrm{O}$ atoms at the surface when cleaving the crystal.

\section{Formation of neutral oxygen defects on the $\mathrm{WO}_{3}$ surface}

In this section the formations of neutral oxygen point defects at the surface are studied. We first focus on the convergence of the vacancy formation energy as a function of the number of planes in the calculation cell. Indeed as shown in our previous study in the bulk [12], an oxygen vacancy formation at the surface is expected to induce significant structural relaxation effects. Finally we show the $n(p)$-doping effect of the oxygen vacancy (oxygen adatom) on the electronic structure of $\mathrm{WO}_{3}$. 


\subsection{Formation energies}

Assuming that the formation of an oxygen vacancy $(\mathrm{V})$ at the surface involves dioxygen $\left(\mathrm{O}_{2}\right)$ by mean of the following reaction:

$$
\mathrm{W}_{n} \mathrm{O}_{3 n} \longrightarrow \frac{1}{2} \mathrm{O}_{2}+\mathrm{W}_{n} \mathrm{O}_{3 n-1}
$$

and neglecting entropy, the formation enthalpy, $H_{V}^{f}$, is given by:

$$
H_{f}^{V}=E_{\left(\mathrm{W}_{n} \mathrm{O}_{3 n-1}\right)}+\mu_{O}-E_{\left(\mathrm{W}_{n} \mathrm{O}_{3 n}\right)},
$$

where $E_{\left(\mathrm{W}_{n} \mathrm{O}_{3 n-1}\right)}$ and $E_{\left(\mathrm{W}_{n} \mathrm{O}_{3 n}\right)}$ are the total energies of the supercell with and without an oxygen vacancy, respectively, and $\mu_{\mathrm{O}}$ is the oxygen chemical potential. In the same manner, the formation reaction and the formation enthalpy of an oxygen adatom are given by:

$$
\mathrm{W}_{n} \mathrm{O}_{3 n}+\frac{1}{2} \mathrm{O}_{2} \longrightarrow \mathrm{W}_{n} \mathrm{O}_{3 n+1}
$$

and

$$
H_{f}^{A}=E_{\left(\mathrm{W}_{n} \mathrm{O}_{3 n+1}\right)}-\mu_{\mathrm{O}}-E_{\left(\mathrm{W}_{n} \mathrm{O}_{3 n}\right)},
$$

where $E_{\left(\mathrm{W}_{n} \mathrm{O}_{3 n+1}\right)}$ is the total of the supercell with an oxygen adatom. Since $\mu_{\mathrm{O}}$ depends on the partial pressure of $\mathrm{O}_{2}$, two limiting cases are considered: ( $i$ ) under ambient atmosphere (O-rich condition), $\mu_{\mathrm{O}}$ reaches an upper limit corresponding to the chemical potential of $\mathrm{O}$ atom in $\mathrm{O}_{2} ;(i i)$ under $\mathrm{O}_{2}$ low partial pressure (O-poor condition), $\mu_{\mathrm{O}}$ reaches a lower limit corresponding to the chemical potential of $\mathrm{O}$ atom in $\mathrm{WO}_{3}$ bulk. This leads to the relation:

$$
\mu_{\mathrm{O}_{2}}+\frac{1}{3} H_{f}^{W O_{3}} \leqslant \mu_{\mathrm{O}} \leqslant \mu_{\mathrm{O}_{2}}
$$


where $\mu_{\mathrm{O}_{2}}$ is half of the total energy of the triplet state $(S=1)$ of $\mathrm{O}_{2}$,

$$
\mu_{\mathrm{O}_{2}}=\frac{1}{2} E_{\left(\mathrm{O}_{2}\right)}
$$

The formation enthalpy of $\mathrm{WO}_{3}$ is given by:

$$
H_{f}^{W O_{3}}=E_{\left(\mathrm{WO}_{3}\right)}^{b u l k}-\frac{3}{2} E_{\left(\mathrm{O}_{2}\right)}-E_{(\mathrm{W})}^{b u l k} .
$$

In this equation $E_{\left(\mathrm{WO}_{3}\right)}^{\text {bulk }}$ is the total energy of the monoclinic $\gamma-\mathrm{WO}_{3}$ and $E_{(\mathrm{W})}^{\text {bulk }}$ is the total energy of the bcc phase of $\mathrm{W}$.

\subsection{Oxygen vacancy formation}

Let us measure the elastic effects due to the finite size of the slab along the perpendicular direction to the surface. The formation enthalpy, $H_{f}^{V}$, is calculated by varying the number of $\mathrm{WO}$ planes from 2 to 8 . Note that in these calculations the oxygen vacancy concentration at the surface, $\mathrm{C}_{V}$, amounts to $1 / 2$ which corresponds to a high concentration. Indeed oxygen vacancies form rows which alternate with oxygen rows along [100] and [010] directions, as schematized on figure 4. This kind of extended defects has been experimentally observed by STM under ultra high vacuum [22]. This

corresponds to O-poor conditions with $\mu_{\mathrm{O}}=\mu_{\mathrm{O}_{2}}+1 / 3 H_{f}^{W O_{3}}$. The translation parameters parallel to the surface correspond to those of the monoclinic phase. For $n=24$ (i.e. slab with 6 WO planes), $H_{f}^{V}$ converges to the value of $-1.4 \mathrm{eV}$ using LDA (see figure 3 ).

When the $\mathrm{W}_{16} \mathrm{O}_{48}$ supercell is doubled along the directions parallel to the surface, $\mathrm{C}_{V}$ decreases from $1 / 2$ to $1 / 8$. Now the oxygen vacancy is surrounded by oxygen atoms in all directions as shown on figure 4 and the interaction between point defects is weakened. Due to this dilution, the formation en- 


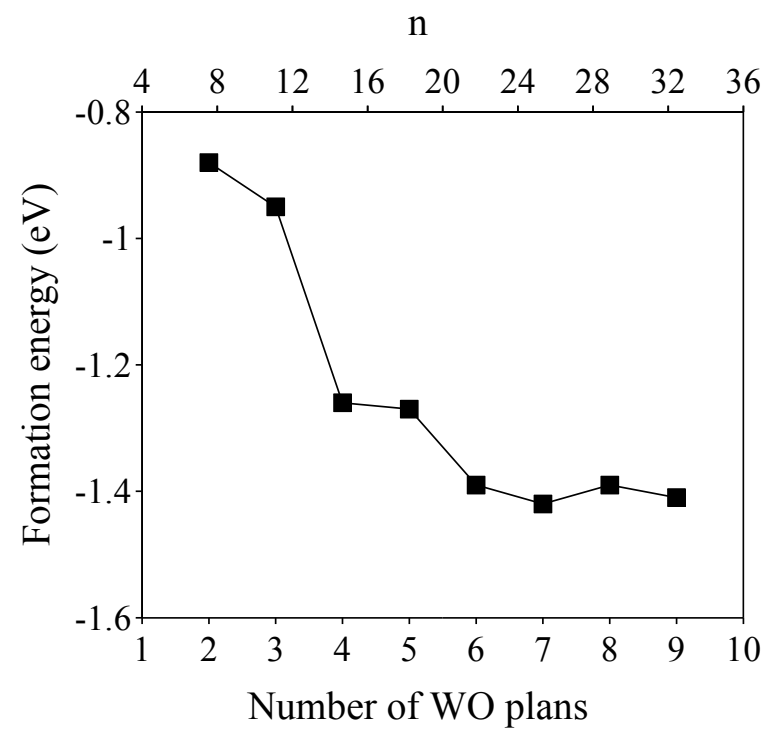

Figure 3: Formation enthalpy of an oxygen vacancy, $H_{f}^{V}$, given in $\mathrm{eV}$, under $\mathrm{O}$-poor condition $\left(\mu_{\mathrm{O}}=\mu_{\mathrm{O}_{2}}+\frac{1}{3} H_{f}^{W O_{3}}\right)$. Lower abscissa: as a function of the number of WO planes. Upper abscissa: as a function of the number $n$ of $\mathrm{W}$ atoms in the cell. LDA results.

thalpy of an oxygen vacancy calculated in the $\mathrm{W}_{64} \mathrm{O}_{192}$ cell decreases from -1.40 to $-1.65 \mathrm{eV}$ under O-poor condition and from 1.70 to $1.45 \mathrm{eV}$ under O-rich condition, within the LDA.

The total density of electronic states $g(\varepsilon)$ is represented on figure 5 for the clean surface and for the surface with a concentration of vacancies $\mathrm{C}_{V}=1 / 8$. We notice that the oxygen vacancy acts as a $n$-doping: the bottom of the conduction band, which mainly consists in $5 d$ states of $\mathrm{W}$, is partially filled. As already mentioned for the bulk [12], this results from the shift of the Fermi level towards higher energies. Due to the underestimate of the band gap (1 eV 


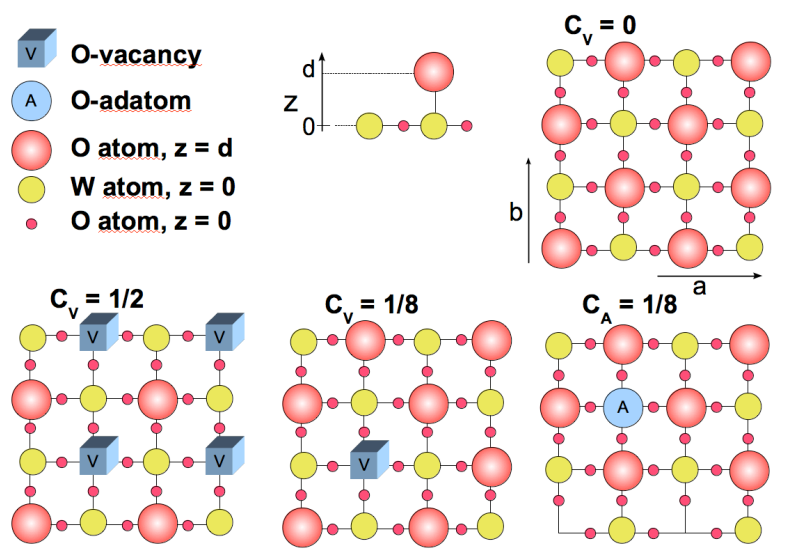

Figure 4: Schematic representation of the surface for oxygen vacancy concentration $\mathrm{C}_{V}=0$ (stoichiometric surface), $\mathrm{C}_{V}=1 / 2$ and $\mathrm{C}_{V}=\mathrm{C}_{A}=1 / 8$. Tungsten atoms (yellow circles) and oxygen atoms (small red circles) belong to the WO plane at $z=0$; surface oxygen atoms (large circles colored in red gradient) and adatom (large bleu circle) are located at $\mathrm{d} \simeq 1.7 \AA$.

instead of $2.5 \mathrm{eV}$ in the bulk), the $n$-doping effect of oxygen vacancies is certainly overestimated. But as discussed hereafter, our results are sufficiently accurate in order to interpret qualitatively the resistivity measurements.

\subsection{Oxygen adatom formation}

The formation of adatom under O-rich condition $(2.70 \mathrm{eV}$ in LDA and $2.42 \mathrm{eV}$ in GGA) requires more energy than the formation of oxygen vacancy independently of the choice of the exchange-correlation functional. In comparison with the vacancy formation, the formation of an oxygen adatom at the stoichiometric (001) surface (see Fig. 4) requires twice energy. This result 

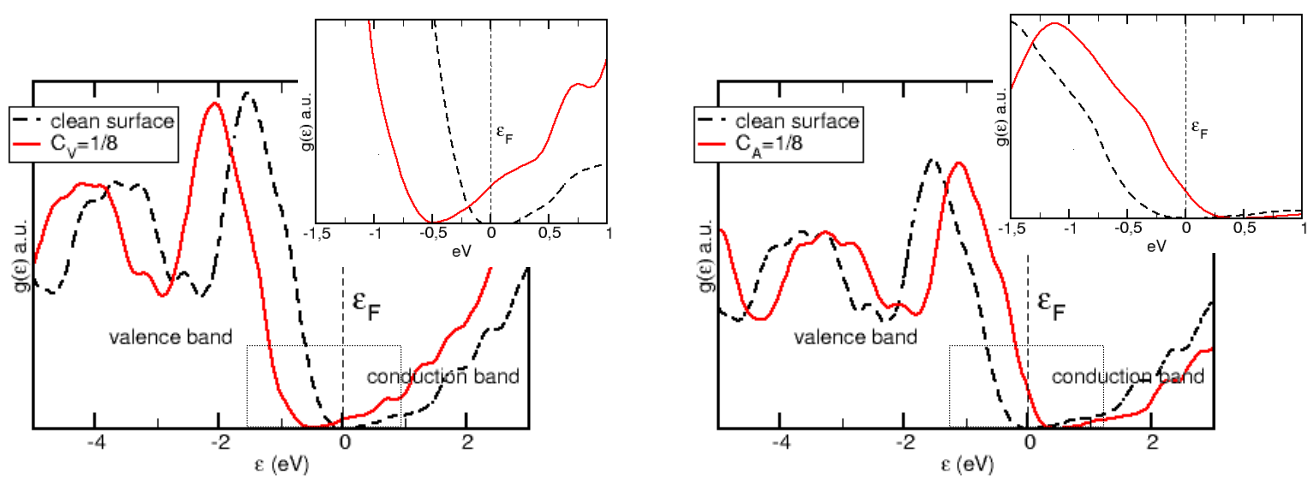

Figure 5: Total density of electronic states $g(\varepsilon)$. Left: comparison between clean surface (dashed line) and surface with vacancy concentration $\mathrm{C}_{V}=1 / 8$. Right: comparison between clean surface (dashed line) and surface with adatom concentration $\mathrm{C}_{A}=1 / 8$. The density of states are obtained from a GGA calculation in the supercell containing $n=64 \mathrm{~W}$ atoms. In all cases the Fermi level, $\varepsilon_{F}$, is chosen as reference energy $(0 \mathrm{eV})$.

is in agreement with experiments for which it is usually observed that $\mathrm{WO}_{3}$ is sub-stoichiometric in oxygen independently of the oxygen partial pressure $[8,23,24,25]$. The total density of electronic states $g(\varepsilon)$ is represented on figure 5 for the clean surface and for the surface with a concentration of adatoms $\mathrm{C}_{A}=1 / 8$. In contrast with the $n$-doping effect due to the vacancy, a $p$-doping is observed when an adatom is created: the top of the valence band which consists mainly in $2 p$ states of $\mathrm{O}$ is partially emptied, leading to a shift of the Fermi level towards lower energies.

\subsection{Effect of the $\mathrm{O}_{2}$ partial pressure}

Let us take into account the effect of the partial pressure of oxygen at the surface on the formation enthalpy of oxygen vacancy and adatom, by plotting $H_{f}^{V}$ and $H_{f}^{A}$ as a function of $\mu_{\mathrm{O}}-\mu_{\mathrm{O}_{2}}$, the relative chemical potential of $\mathrm{O}$ 


\begin{tabular}{l|c|c||c|c}
\hline & \multicolumn{2}{|c||}{ LDA } & \multicolumn{2}{c}{ GGA } \\
\hline$H_{f}^{W O_{3}}$ & \multicolumn{2}{|c||}{-9.30} & \multicolumn{2}{c}{-7.92} \\
\hline$\mu_{\mathrm{O}}$ & $\mu_{\mathrm{O}_{2}}$ & $\mu_{\mathrm{O}_{2}}+\frac{1}{3} H_{f}^{W O_{3}}$ & $\mu_{\mathrm{O}_{2}}$ & $\mu_{\mathrm{O}_{2}}+\frac{1}{3} H_{f}^{W O_{3}}$ \\
\hline$H_{f}^{V}$ & 1.45 & -1.65 & 1.28 & -1.36 \\
$H_{f}^{A}$ & 2.70 & 5.80 & 2.42 & 5.06 \\
\hline \hline
\end{tabular}

Table 3: Formation enthalpies (given in eV) of the $\mathrm{WO}_{3}$ bulk $\left(H_{f}^{W O_{3}}\right)$, oxygen vacancy $\left(H_{f}^{V}\right)$ and adatom $\left(H_{f}^{A}\right)$, under O-rich $\left(\mu_{\mathrm{O}}=\mu_{\mathrm{O}_{2}}\right)$ and O-poor $\left(\mu_{\mathrm{O}}=\mu_{\mathrm{O}_{2}}+\frac{1}{3} H_{f}^{W O_{3}}\right)$ conditions. Calculations are performed for $\mathrm{W}_{64} \mathrm{O}_{192}$ supercell within the LDA and the GGA.

atom. The value of $H_{f}^{W O_{3}}$ and the values of $H_{f}^{V}$ and $H_{f}^{A}$ are reported on table 3 for the two limit conditions of $\mu_{\mathrm{O}}$. The energy differences between LDA and GGA do not exceed twenty percent and the interpretation of the results is qualitatively the same. Under O-rich condition the values of $H_{f}^{V}$ (1.45 eV within the LDA and $1.28 \mathrm{eV}$ within the GGA) are almost half of those obtained in the bulk (3.39 eV and $2.87 \mathrm{eV}$ respectively). Figure 6 represents the linear variation of the formation enthalpies of oxygen defects with respect to $\mu_{\mathrm{O}}-\mu_{\mathrm{O}_{2}}$ for a constant concentration of defect $C_{V}=C_{A}=$ $1 / 8$. For any value of $\mu_{\mathrm{O}}, H_{f}^{V}$ is found to be lower than $H_{f}^{A}$ (table 6). This indicates that the oxygen vacancy is the dominant defect at the surface. When the partial pressure of oxygen decreases, $H_{f}^{V}$ decreases, while $H_{f}^{A}$ increases.

As observed experimentaly, lowering the oxygen partial pressure favors the formation of oxygen vacancy $[8,24,25]$. Thus under O-poor conditions the formation enthalpy of oxygen vacancy is exothermic $(-1.65 \mathrm{eV}$ within the LDA) whereas the energy cost for the formation of an adatom becomes 

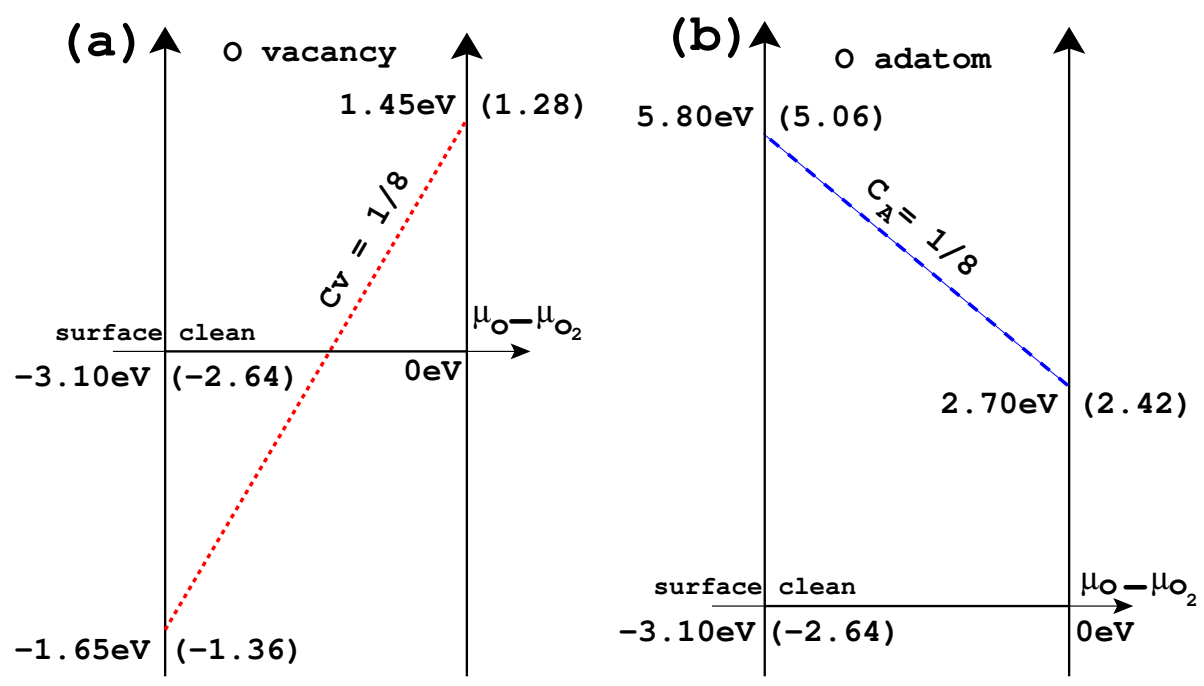

Figure 6: Formation enthalpies in eV: (a) for an oxygen vacancy and (b) for an oxygen adatom as function of $\mu_{\mathrm{O}}-\mu_{\mathrm{O}_{2}}$, the relative chemical potential of $\mathrm{O}$ atom. $\mu_{\mathrm{O}_{2}}$ is the chemical potential in $\mathrm{O}_{2}$ molecule (O-rich condition). The lower limit of $\mu_{O}-\mu_{O_{2}}$ corresponds to the chemical potential of $\mathrm{O}$ in $\mathrm{WO}_{3}$ (O-poor condition). The reference energy is the total energy of the clean surface, i.e. without any defect. Comparison between LDA and GGA (GGA results are shown in brackets).

extremely large $(+5.70 \mathrm{eV}$ within LDA $)$.

\section{Conclusion}

Among the reconstructed (001) surfaces of the monoclinic $\gamma-\mathrm{WO}_{3}$ phase, the $(\sqrt{2} \times \sqrt{2}) \mathrm{R} 45^{\circ}$ reconstruction is found to be the most stable for the reconstructed (001) surfaces in the cubic phase [11]. The detailed study of the neutral point defects (vacancy and adatom) reveals that the difference in the formation enthalpies do not exceed twenty percent between LDA and GGA. By introducing a thermodynamic model, the partial pressure of oxygen is taken into account by the variation of the chemical potential $\mu_{\mathrm{O}}$. We highlight 
that the oxygen vacancy is the dominant defect whatever the oxygen partial pressure. Between the two extrem limits - from O-rich condition to O-poor condition - the formation enthalpy of an oxygen adatom rises from $2.70 \mathrm{eV}$ to $5.50 \mathrm{eV}$ within the LDA. On the other hand, the formation enthalpy of an oxygen vacancy decreases from $1.45 \mathrm{eV}$ to $-1.65 \mathrm{eV}$. This indicates that the well-known sub-stoichiometry in oxygen of $\mathrm{WO}_{3}$ is favored by decreasing oxygen partial pressure. Moreover point defects modify the doping of the $\mathrm{WO}_{3}$ sensitive layer of gas sensor device. We show that the formation of an oxygen vacancy leads to the filling of the bottom of the conduction band, by increasing the number of negative charge carriers. The formation of an adatom acts in an opposite way, since it leads to the creation of positive charge carriers at the top of the valence band.

All these results can be strongly correlated to the experiments [24, 25, 26], which have shown that the electrical resistivity of $\mathrm{WO}_{3}$ depends not only on the temperature but also on the oxygen partial pressure. So at room temperature, the resistivity rises by one order of magnitude when the air pressure increases from 0.01 to 1 bar. Under vacuum after annealing at $673 \mathrm{~K}$, the conductivity is almost constant with temperature, $5(\Omega \mathrm{cm})^{-1}$ approximatively. So, the concentration of vacancies reaches a maximum and consequently, the conductivity too. These characteristics indicate that the $\mathrm{WO}_{3}$ thin film has a metallic behaviour. This can be interpreted by our calculations as follows. Thermodynamically, when the partial pressure of oxygen decreases, the formation enthalpy of an oxygen vacancy, $H_{f}^{V}$, decreases. Within the Boltzmann description, the concentration of vacancy, $C_{V}$, depends on the ratio $-H_{f}^{V} / k T$. Thus, when increasing the temperature or when decreasing 
the oxygen partial pressure, the number of negative charge carriers rises due to the increase of $C_{V}$. Under vacuum, our thermodynamic model shows that the vacancy formation enthalpy from a stoichiometric surface is exothermic. Therefore the vacancy concentration should reach a maximum (probably $1 / 2$ as observed in reference [22]). Consequently the number of negative charge carriers and the electrical conductivity reach a maximum. To conclude, the energy quantities deduced from ab initio simulations can be introduced in analytical models developed in order to quantitavely describe the sensor response as a function of partial pressure of toxic gases [8].

\section{Acknowledgements}

The authors are very grateful to Roland Hayn, Pierre Müller, Thomas Frisch and Jacques Guérin for numerous helpful discussions. The calculations were supported by the 'Centre Informatique National de l'Enseignement Supérieur' (CINES-France).

\section{References}

[1] K. Miyake, H. Kaneko, M. Sano, N. Suedomi, J. Appl. Phys. 55(7) (1984) 2747.

[2] E. Washizu, A. Yamamoto, Y. Abe, M. Kawamura, K. Sasaki, Solid State Ionics 165 (2003) 175.

[3] H. A. Wriedt, Bulletin of Alloy Phase Diagrams 10 (4) (1989) 368.

[4] E. Llobet, G. Molas, P. Molinás, J. Calderer, X. Vilanova, J. Brezmes, 
J. E. Sueiras, X. Correig, Journal of The Electrochemical Society 147 (2) (2000) 776 .

[5] X. Wang, N. Miura, N. Yamazoe, Sensors and Actuators B 66 (2000) 74 .

[6] C. Cantalini, W. Wlodarski, Y. Li, M. Passacantando, S. Santucci, E. Comini, G. Faglia, G. Sberveglieri, Sensors and Actuators B 64 (2000) 182.

[7] D. E. Williams, S. R. Aliwell, K. F. E. Pratt, D. J. Caruana, R. L. Jones, R. A. Cox, G. M. Hansford, J. Halsall, Meas. Sci. Technol. 13 (2002) 923.

[8] J. Guérin, K. Aguir, M. Bendahan, C. Lambert-Mauriat, Sensors and Actuators B 104 (2005) 289.

[9] S. Gomri, J. L. Seguin, J. Guérin, K. Aguir, Sensors and Actuators B 114 (2006) 451.

[10] J. Oviedo, M. J. Gillan, Surface Science 490 (2001) 221.

[11] P. M. Oliver, S. C. Parker, R. G. Egdell, F. H. Jones, J. Chem. Soc., Faraday Trans. 92 (1996) 2049.

[12] C. Lambert-Mauriat, V. Oison, J. Phys.: Condens. Matter 18 (2006) 7361.

[13] P. Ordejón, E. Artacho, J. M. Soler, Phys. Rev. B 53 (1996) R10441. 
[14] J. M. Soler, E. Artacho, J. D. Gale, A. Garcia, J. Junquera, P. Ordejón, D. Sánchez-Portal, J. Phys.: Condens. Matter 14 (2002) 2745.

[15] D. M. Ceperley, B. J. Alder, Phys. Rev. Lett. 45 (1980) 566.

[16] J. P. Perdew, K. Burke, M. Ernzerhof, Phys. Rev. Lett. 77 (1996) 3865.

[17] N. Troullier, J. L. Martins, Phys. Rev. B 43 (1991) 1993.

[18] O. F. Sankey, D. J. Niklewski, Phys. Rev. B 40 (1989) 3979.

[19] B. Loopstra, H. Rietveld, Acta Crystallographica b 25 (1969) 1420.

[20] I. N. Yakovkin, M. Gutowski, Surface Science 610 (2007) 1481.

[21] R. A. Dixon, J. J. Williams, D. Morris, J. Rebane, F. H. Jones, R. G. Egdell, S. W. Downes, Surface Science 399 (1998) 199.

[22] F. H. Jones, K. Rawlings, J. S. Foord, R. G. Egdell, B. . M. R. Wanklyn, S. C. Parker, P. M. Oliver, Surface Science 359 (1996) 107.

[23] J. M. Berak, M. J. Sienko, J. Solid State Chem. 2 (1970) 109.

[24] K. Aguir, C. Lemire, D. Lollman, Sensors and Actuators B 84 (2002) 1.

[25] M. Gillet, K. Aguir, C. Lemire, E. Gillet, K. Schierbaum, Thin Solid Films 467 (2004) 239.

[26] M. Gillet, C. Lemire, E. Gillet, K. Aguir, Surface Science 532-535 (2003) 519. 